\title{
Engaging Patients via Mobile Phone Technology to Assist Follow-Up After Hospitalization in Quito, Ecuador
}

Julie Maslowsky, M.A., M.S.W., ${ }^{1}$ Bina Valsangkar, M.D., M.P.H., ${ }^{2}$ Jennifer Chung, B.S., ${ }^{3}$ Jennifer Rasanathan, M.D., M.P.H., ${ }^{3}$ Freddy Trujillo Cruz, M.D., ${ }^{4}$ Marco Ochoa, M.D., ${ }^{4}$

Monica Chiriboga, M.D., ${ }^{5}$ Fernando Astudillo, M.D., ${ }^{6}$ Michele Heisler, M.D., M.P.A., ${ }^{7,8}$ and Sofia Merajver, M.D., Ph.D. ${ }^{7,9,10}$

${ }^{1}$ Department of Psychology and ${ }^{9}$ Center for Global Health, University of Michigan, Ann Arbor, Michigan.

${ }^{2}$ Children's National Medical Center, Washington, D.C.

${ }^{3}$ University of Michigan Medical School, Ann Arbor, Michigan.

${ }^{4}$ Hospital Enrique Garces, Quito, Ecuador.

${ }^{5}$ Guamani Health Center, Quito, Ecuador.

${ }^{6}$ Central University of Ecuador, Quito, Ecuador.

${ }^{7}$ Department of Internal Medicine, University of Michigan Health System, Ann Arbor, Michigan.

Departments of ${ }^{8}$ Health Behavior and Health Education and

${ }^{10}$ Epidemiology, University of Michigan School of Public Health, Ann Arbor, Michigan.

\begin{abstract}
Objective: Disease management following hospital discharge is difficult in most low-resourced areas, posing a major obstacle to health equity. Although mobile phones are a ubiquitous and promising technology to facilitate healthcare access, few studies have tested the acceptability and feasibility of patients themselves using the devices for assisting linkages to healthcare services. We hypothesized that patients would use mobile phones to help manage postdischarge problems, if given a communication protocol. We developed a mobile phone-based program and investigated its acceptability and feasibility as a method of delivering posthospitalization care. Subjects and Methods: A consecutive cohort of adult patients in a public hospital in Quito, Ecuador was enrolled over a 1-month period. A hospital-based nurse relayed patients' discharge instructions to a community-based nurse. Patients corresponded with this nurse via text messaging and phone calls according to a protocol to initiate and participate in follow-up. Results: Eighty-nine percent of eligible patients participated. Ninety-seven percent of participants completed at least one contact with the nurse; $81 \%$ initiated contact themselves. Nurses completed 262 contacts with 32 patients, clarifying discharge instructions, providing preventive education, and facilitating clinic appointments. By this method, 87\% of patients were successfully linked to follow-up appointments. Conclusions: High levels of patient participation and successful delivery of followup services indicate the mobile phone program's acceptability and
\end{abstract}

feasibility for facilitating posthospitalization follow-up. Patients actively used mobile phones to interact with nurses, enabling the provision of posthospitalization medical advice and facilitate community-based care via mobile phone.

Key words: technology, telecommunications, telehealth

\section{Introduction}

T he need for posthospitalization follow-up poses a special challenge for the healthcare infrastructures of low- and mid-resourced areas, which often struggle to meet the demand for inpatient services. Leveraging active patient participation and exploiting new communication technologies are strategies amenable to overcoming this challenge. With 5.3 billion users worldwide, mobile phone-based technologies are a promising vehicle for innovation in global health. ${ }^{1}$ Major advantages of mobile phones include their relatively low cost and near-ubiquity in rural and low-income areas. In 2010, 90\% of the world's population had access to a mobile network, with 68\% penetration in the developing world. ${ }^{2}$ In Ecuador, mobile phone penetration is estimated at $95 \% .^{3}$

The widespread use of mobile phone technology in the developing world has led to proliferation of mobile phone-based programs to address acute and chronic health conditions and provision of primary healthcare. One area of success has been in providing appointment reminders via automated text messages, which have been associated with significant improvements in appointment attendance in several developing countries. ${ }^{4-6}$

To date, most mobile phone-based global health intervention programs have relied on unidirectional communications with patients, such as automated text messages. ${ }^{7}$ Although effective for some purposes, including appointment and medication reminders, automated methods do not address the range of patients' needs following discharge from tertiary care, nor are they part of an interactive patient-healthcare provider partnership. Several studies have moved beyond unidirectional communication in mobile phonebased programs. When provided with the mobile phone number of a community physician and invited to call for medical consultation, a majority of Indian patients rated the system as helpful for managing their conditions. ${ }^{8}$ In Korea, investigators used short message service to monitor and subsequently improve glycosylated hemoglobin levels in diabetic patients. ${ }^{9}$ These and other studies have begun to demonstrate the power of mobile phone technology to improve outpatient follow-up care, ${ }^{10-12}$ although rigorous evaluations of their impacts are lacking. ${ }^{13,14}$ Nonetheless, the early promise of mobile phones in healthcare provision in the developing world has 
resulted in a call for increasing such programs and rigorously evaluating their potential for impact. ${ }^{13,15}$ Latin American countries are an auspicious venue for mobile phone-based programs given the combination of high mobile phone network penetration and demand for healthcare services that far exceeds the systems' capacity. The current program providing mobile phone-based follow-up care was developed in response to the Ecuadorian Ministry of Public Health's identification of poor patient adherence to hospital discharge instructions and inadequate follow-up in the community as significant obstacles to achieving good health outcomes.

A key first step in developing the potential of mobile phones in global public health is to establish the feasibility of the specific technological interventions in different contexts and adapt these to meet local needs and practices. Such studies are essential for building effective mobile phone-based health programs adapted to country, culture, and political and economic climate. As there are currently no acceptability or feasibility data on patient-centered mobile technologies in Latin America, the current study sought to measure the acceptability and feasibility of a mobile phone-based public health program in an underserved, low-income, semiurban community.

Ecuador is a lower-middle income country with a GDP per capita of $\$ 4,013$ in 2010. ${ }^{16}$ Southern Quito is an area of concentrated poverty, although with extensive mobile phone penetration. The overall objective of this study was to examine the feasibility and patient acceptability of using mobile voice and text communication with an on-call nurse to improve adherence to discharge instructions and facilitate follow-up care following hospital discharge.

Three research questions were addressed: (1) Is the mobile phone program acceptable to patients? (2) Is the program a feasible method of providing patient-centered feedback to assist posthospitalization follow-up? (3) In what manner do patients use mobile phones? For purposes of the current study, acceptability was defined as the program being satisfactory and agreeable to the patients, as measured by patients' willingness to enroll and participate in the program. Feasibility was defined as the possibility for the program to be implemented successfully in the current setting, as measured by patients' ability to use mobile phones in the suggested manner without difficulties and nurses' ability to provide follow-up via mobile phone.

\section{Subjects and Methods STUDY POPULATION}

Patients were recruited from a large public referral hospital in Quito that serves a catchment population of approximately 1 million. Patients eligible for this study were those referred by health clinics within one specific geographic health zone in southern Quito, representing a catchment area of approximately 89,000 people. Inclusion criteria were as follows: Patient was at least 18 years old, spoke Spanish, and was referred from the target health zone.
A consecutive cohort of all patients meeting these criteria who were admitted to the hospital during the 1-month study period was targeted for enrollment; the cohort comprised 39 patients. Recruitment was not limited by medical condition or health status, as the aim was to test uptake of the technology in a general patient population. Of 39 patients invited to enroll, 35 (89.7\%) consented to participate. Of those, 32 were discharged from the hospital during the study period and were eligible to complete posthospitalization follow-up. Therefore, these 32 patients made up the evaluable sample for the current report (Fig. 1).

\section{PROCEDURES}

All study procedures were approved by the Institutional Review Board of the University of Michigan and the Ethics Committee of the Ministry of Public Health of Ecuador. All patients enrolled in the study provided informed consent. Once enrolled, patients who did not own a mobile phone were provided with one, and patients who already owned a mobile phone were given credit to use the phone.

Typical discharge procedures in the hospital studied consist of verbal instructions issued by the attending physician and a written plan of follow-up that patients are instructed to take to the community clinic when continuing care. Patients are expected to initiate their own community follow-up care; there is no system of communication between the hospital and community health clinic regarding their mutual patients. The current project created such a system via patient-initiated, patient-centered communication with nurses devoted to follow-up care. Two nurses, one based in the hospital and one in the community health center, were on duty every day from 8 a.m. to 5 p.m., to facilitate continuity of care as the patient transitioned from the hospital to community-based health clinics. These nurses did not receive any special training besides that needed for them to be familiar with the study design. The hospital-based

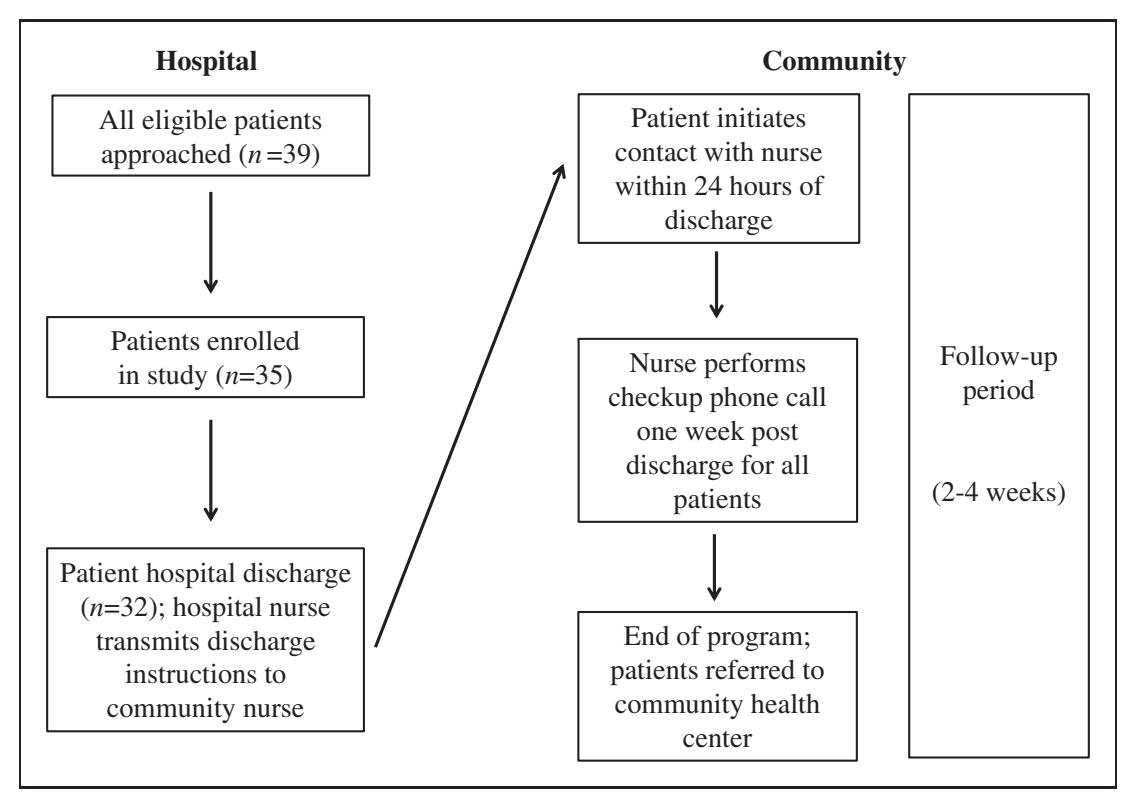

Fig. 1. Summary of patient enrollment and follow-up procedures. 


\section{MOBILE PHONE FOLLOW-UP IN QUITO, ECUADOR}

\begin{tabular}{|c|c|}
\hline CODE & MESSAGE \\
\hline \multirow[t]{2}{*}{1} & $\begin{array}{l}\text { I am fine. I understand my discharge and medication instructions. } \\
\text { I do not need help now. }\end{array}$ \\
\hline & $\begin{array}{l}\text { Estoy bien. Entiendo las instrucciones del alta y de los } \\
\text { medicamentos. No necesito ayuda ahora. }\end{array}$ \\
\hline \multirow[t]{2}{*}{2} & $\begin{array}{l}\text { I am fine, but I do not understand my discharge and/or medication } \\
\text { instructions. }\end{array}$ \\
\hline & $\begin{array}{l}\text { Estoy bien, pero no entiendo las instrucciones del alta y/o de los } \\
\text { medicamentos. }\end{array}$ \\
\hline \multirow[t]{2}{*}{3} & I am worried about my health. I want to talk to the nurse. \\
\hline & Estoy preocupado/a por mi salud. Quiero hablar con la enfermera. \\
\hline \multirow[t]{2}{*}{4} & I am sick. I am returning to the hospital. \\
\hline & Estoy enfermo/a. Regreso al hospital. \\
\hline
\end{tabular}

nurse attended each patient's discharge, noted all instructions given by the doctor (medication regimen, follow-up appointments needed, and self-care that should be completed), and communicated these instructions to the community-based nurse.

Patients were instructed to send a text message to the nurse within $24 \mathrm{~h}$ of discharge to relay their health status and initiate follow-up. They were further informed that they could contact the nurse at any time with questions regarding their health status, medications, or discharge instructions and for assistance scheduling a follow-up medical appointment. Patients who did not send a text message within $24 \mathrm{~h}$ were called by the nurse to initiate follow-up. Length of follow-up ranged from 1 to 4 weeks, with all patients receiving a minimum of 1 week. At the end of the program, all patients completed a five-item survey regarding their satisfaction with the program and how they would have sought follow-up care if they had not participated in this program.

Before discharge, all patients were trained by research assistants (advanced Ecuadorian medical students) to use a simple numerical menu to transmit their health status via text message (Table 1). A numerical menu was used to streamline communication between the patient and the nurse and to objectively evaluate adherence to the study guidelines; patients were asked to use only the codes provided in all of their texts. This menu was affixed to each patient's mobile phone, along with emergency contact numbers for the hospital and community health centers. Patients were instructed to call these numbers, rather than the on-call nurse, in case of an emergency.

Codes 1-3 triggered a phone call from the community-based nurse. Code 4 initiated monitoring by the nurse to determine whether the patient was re-admitted to the hospital, in which case follow-up was re-initiated at discharge. In addition to text messages, patients were free to call the nurse with questions or concerns. In response to patient text messages and phone calls, the community-based nurse scheduled follow-up appointments at the community health center, provided reminders of discharge and medication instructions, and conducted preventive education as appropriate. Patients also received a 1-week follow-up call after hospital discharge to monitor their clinical status (Fig. 1).

\section{Results}

Patients' ages ranged from 18 and 60 years, averaging 29 years. The majority $(75.0 \%)$ of patients were female. The most common reason for hospitalization was an obstetric condition (71.9\% of patients), including nine vaginal births, seven cesarean sections, five miscarriages, and two female sterilizations. Other reasons for hospitalization included surgery, acute illness, injury, and psychiatric illness. Characteristics of enrolled patients are presented in Table 2.

\section{PATIENT PARTICIPATION}

The first set of analyses was conducted on the patient level, assessing patients' access to and ability to use mobile phones and their level of participation in the program (Table 3). The majority of patients $(62.5 \%)$ used their own mobile phones while participating in the study; the remaining patients were provided with a mobile phone to enable participation. All patients received training prior to discharge on basic mobile phone operation, sending text messages, and using program-specific text codes. All patients reported understanding of the text codes and sent a successful practice message during training. Following training, most reported that they did not need additional help using the phone (59.4\%), or that they might need help but someone in the home could help if needed (37.5\%). One patient who reported needing help and no one at home to assist received extra training to ensure his confidence using the mobile phone.

Contact was made within $24 \mathrm{~h}$ of discharge for $96.9 \%$ of patients. Eleven patients (34.3\%) made initial contact by sending the nurse a text message as requested. For 20 patients (62.5\%), the format of initial contact was a phone call from the nurse to the patient. One patient was lost to follow-up.

The majority of patients (81.3\%) self-initiated contact with the nurse at some point during the study, by either phone call or text message. Eighteen (56.3\%) patients sent at least one text message to the nurse; 20 (60.6\%) called the nurse at least once.

\section{TYPE AND CONTENT OF PATIENT CONTACTS}

The second set of analyses was conducted at the level of contacts with patients (Table 4). There were 262 nurse-patient contacts, an average of eight per patient. The majority of contacts $(66.4 \%)$ were nurse-initiated calls to patients. Of 87 patient-initiated contacts, 57 (65.5\%) were phone calls, and 30 (34.5\%) were text messages. It is notable that the number of contacts per patient did not vary according to whether the patient was given a mobile phone or used his own $[t(30)=0.92, p>0.05]$, nor did the proportion of patients who initiated a text message $\left[\chi^{2}(1)=0.85, p>0.05\right]$ or phone call $\left[\chi^{2}(1)=0.14, p>0.05\right)$ to the nurse. 
Table 2. Patient Demographic and Clinical Characteristics $(n=32)$

\section{CHARACTERISTIC}

\section{$N(\%)$}

Sex

\begin{tabular}{|c|c|}
\hline Female & $24(75.0)$ \\
\hline Male & $8(25.0)$ \\
\hline \multicolumn{2}{|l|}{ Reason for hospitalization ${ }^{a}$} \\
\hline Obstetric & $23(71.9)$ \\
\hline Surgery (non-obstetric) & $4(15.6)$ \\
\hline Acute illness & $3(9.4)$ \\
\hline Injury & $3(9.4)$ \\
\hline Psychiatric & $1(3.1)$ \\
\hline \multicolumn{2}{|l|}{ Patient's occupation } \\
\hline Homemaker & $16(50.0)$ \\
\hline Manual labor & $5(15.6)$ \\
\hline Service & $5(15.6)$ \\
\hline Skilled trade & $2(6.3)$ \\
\hline Professional & $2(6.3)$ \\
\hline Student & $1(3.1)$ \\
\hline Not reported & $1(3.1)$ \\
\hline \multicolumn{2}{|c|}{ Patient was provided with a cell phone } \\
\hline Yes & $12(37.5)$ \\
\hline No & $20(62.5)$ \\
\hline
\end{tabular}

Has help at home to use cell phone if needed

\begin{tabular}{l|c}
\hline Yes & $12(37.5)$ \\
\hline No & $1(3.1)$ \\
\hline Not needed & $19(59.4)$ \\
\hline Age (years) [mean (SD)] & $29.1(10.1)$ \\
\hline $\begin{array}{l}\text { Length of hospitalization (days) [mean } \\
\text { (SD)] }\end{array}$ & $4.3(3.1)$ \\
\hline
\end{tabular}

${ }^{a}$ Multiple reasons for hospitalization were noted for some patients.

SD, standard deviation.

Text messages. The content of patients' text messages is described in Table 4. The nurse responded to $100 \%$ of text messages with a call or, in one case, an in-person contact at the community clinic.

Phone calls. In total, 231 phone calls were completed between patients and the nurse. The most common topics of conversation were as follows: The nurse monitoring the patient's health status, providing preventive education, facilitating clinic appointments, and
Table 3. Patient Participation $(n=32)$ PARTICIPATION $N(\%)$

Initial patient contact made within $24 \mathrm{~h}$

\begin{tabular}{c|c}
\hline Yes & $31(96.9)$ \\
\hline No & $1(3.1)$ \\
\hline
\end{tabular}

Format of initial contact

\begin{tabular}{l|c}
\hline Text sent by patient & $11(34.4)$ \\
\hline Phone call by nurse & $20(62.5)$ \\
\hline None & $1(3.1)$ \\
\hline
\end{tabular}

Patient ever sent text to nurse

\begin{tabular}{l|c}
\hline Yes & $18(56.3)$ \\
\hline No & $14(43.8)$ \\
\hline
\end{tabular}

Patient ever called nurse

\begin{tabular}{l|l}
\hline Yes & $20(62.5)$ \\
\hline No & $12(37.5)$ \\
\hline
\end{tabular}

Nurse ever called patient

\begin{tabular}{l|c}
\hline Yes & $32(100.0)$ \\
\hline No & $0(0.0)$ \\
\hline Patient ever initiated contact with nurse \\
\hline Yes & $26(81.3)$ \\
\hline No & $6(18.8)$ \\
\hline
\end{tabular}

Nurse attempted 1-week follow-up call

\begin{tabular}{c|c}
\hline Yes & $32(100.0)$ \\
\hline No & $0(0.0)$ \\
\hline
\end{tabular}

Nurse completed 1-week follow-up call

\begin{tabular}{l|c}
\hline Yes & $30(93.8)$ \\
\hline No & $2(6.3)$ \\
\hline Doctor prescribed follow-up clinic visit \\
\hline Yes & $31(96.8)$ \\
\hline No & $1(3.2)$ \\
\hline
\end{tabular}

If prescribed, patient attended follow-up visit

\begin{tabular}{l|c}
\hline Yes & $27(87.1)$ \\
\hline No & $2(6.5)$ \\
\hline Unable to ascertain & $2(6.5)$ \\
\hline
\end{tabular}

Patient's end of program status

\begin{tabular}{l|c}
\hline Successfully treated and case closed & $28(87.5)$ \\
\hline Required no follow-up treatment & $3(9.4)$ \\
\hline Lost to follow-up & $1(3.1)$ \\
\hline
\end{tabular}

continued $\rightarrow$ 
Table 3. Patient Participation $(\boldsymbol{n}=\mathbf{3 2})$ continued CONTACT

$N(\%)$

Patient response: What would you have done without this service?

\begin{tabular}{l|c}
\hline Returned to hospital & $11(39.3)$ \\
\hline Gone to community clinic & $8(28.6)$ \\
\hline Tried to treat myself & $5(17.9)$ \\
\hline Nothing & $2(7.1)$ \\
\hline Don't know & $2(7.1)$ \\
\hline
\end{tabular}

${ }^{a} n=28$ respondents to end-of-program survey.

clarifying hospital discharge instructions. Nurses also provided emotional support and fielded questions regarding health problems unrelated to the hospitalization. Less common topics of discussion included infant vaccinations and referrals to outside facilities (e.g., pharmacies). The prevalence of each topic of conversation did not significantly differ according to whether the contact was initiated by the nurse or patient, with the exception of "problem not related to discharge diagnosis," which occurred more often during a patient- than a nurse-initiated contact $[t(234)=5.54, p<0.001]$.

\section{FACILITATING FOLLOW-UP CARE IN THE COMMUNITY}

Nurses attempted 1-week follow-up calls with all 32 patients; 30 (93.8\%) were completed. Upon discharge, 31 (96.8\%) patients were prescribed a follow-up clinic visit. Twenty-seven (87.1\%) patients reported completing these visits, 2 (6.5\%) did not attend their visit, and $2(6.5 \%)$ could not be reached to verify their attendance.

Twenty-eight patients completed the end-of-program survey. These patients were asked what they would have done to receive follow-up care if the mobile phone option were not available. Eleven (39.3\%) stated they would have returned to the hospital, 8 (28.6\%) would have gone to a community clinic, 5 (17.9\%) would have attempted self-treatment, 2 (7.1\%) would have done nothing, and 2 (7.1\%) were unsure.

\section{Discussion}

This study addressed a significant self-identified need within Ecuador's overburdened public health system to improve the efficiency and efficacy of existing health services by exploring use of a patient-centered self-monitoring system for discharged hospitalized patients. The primary objective of this study was to examine the program's acceptability and feasibility. Given the high degree of patient participation in the study and both the variety and amount of follow-up services provided as a result of patients' use of the mobile phone program, the results suggest that the use of mobile phones for follow-up in this setting is both acceptable and feasible and warrants further detailed study.

The program's acceptability was indicated by high rates of patient participation: $96.9 \%$ of enrolled patients actively participated

\section{Table 4. Type and Content of Nurse-Patient Contacts} CONTACT $N(\%)$

All contacts $(n=262)$

Type of contact

\begin{tabular}{l|c}
\hline Nurse-initiated phone call & $174(66.4)$ \\
\hline Patient-initiated phone call & $57(21.8)$ \\
\hline Patient-initiated text message & $30(11.5)$ \\
\hline Home visit & $1(0.4)$ \\
\hline
\end{tabular}

Text messages $(n=30)$

Text message code received from patient

\begin{tabular}{l|c}
\hline $1=$ "I am fine" & $15(50.0)$ \\
\hline $\begin{array}{l}2=" I \text { do not understand my medication/discharge } \\
\text { instructions" }\end{array}$ & $1(3.3)$ \\
\hline $3=$ "I am worried about my health" & $3(10.0)$ \\
\hline $4=" I$ am returning to the hospital" & $7(23.3)$ \\
\hline Other & $4(13.3)$ \\
\hline
\end{tabular}

Nurse attempted call following receipt of text

\begin{tabular}{c|c}
\hline Yes & $29(96.7)$ \\
\hline No & $1(3.3)^{2}$ \\
\hline
\end{tabular}

If attempted, call following receipt of text completed successfully?

\begin{tabular}{c|c}
\hline Yes & $29(100.0)$ \\
\hline No & $0(0.0)$ \\
\hline
\end{tabular}

Phone calls $(n=231)^{b}$

Topic of phone call

\begin{tabular}{l|c}
\hline Monitor patient health status & $114(49.4)$ \\
\hline Preventive education & $111(48.1)$ \\
\hline Facilitate a clinic appointment & $109(47.2)$ \\
\hline $\begin{array}{l}\text { Clarify hospital discharge and/or medication } \\
\text { instructions }\end{array}$ & $84(36.4)$ \\
\hline Emotional support & $31(13.4)$ \\
\hline Problem other than discharge diagnosis & $17(7.4)$ \\
\hline Infant vaccinations & $11(4.8)$ \\
\hline Referral to outside health facility & $8(3.5)$ \\
\hline
\end{tabular}

${ }^{a}$ In this case, the nurse saw the patient in person in the clinic rather than replying to the text.

${ }^{b}$ Phone calls include those initiated by either the nurse or the patient.

'Topic of phone call sums to $>100 \%$ because each phone call may have included more than one topic of discussion. 


\section{MASLOWSKY ET AL.}

in the program's services after hospital discharge, and $81.3 \%$ of patients initiated contact with the nurse during the program. Its feasibility was demonstrated by the diversity of services delivered directly and indirectly as a result of the program. The nurses provided substantial clarification regarding hospital discharge and medication instructions, educated patients on prevention and health promotion, and successfully facilitated necessary follow-up appointments in community health centers. Although the program was designed to function via either text messages or phone calls, phone calls were the dominant mode of communication used by patients. Thus, an important lesson of this study is that patients in this setting prefer to initiate contact with a nurse via phone call rather than text message. Also of note is that some patients who did not own a mobile phone were provided with one in order to participate. These patients communicated with the nurse equally as often as those who owned mobile phones, indicating ease of adoptability of the protocol.

As a further measure of patient acceptability, the vast majority of patients reported in the end-of program survey that they would have been willing to use the program even if mobile phone credit was not given, and they would have been willing to pay for costs incurred. Forty percent of participants indicated that they would have sought treatment at the hospital but instead received help from the mobile phone program, rendering the hospital visit unnecessary. Therefore, this study suggests the program's potential to reduce the burden of patient emergency room and hospital visits on the health system.

\section{Conclusions}

Previous studies support the promise of mobile phone-based programs as a means of healthcare delivery and health system improvement. ${ }^{1,4-6,8-12}$ The current study extends this literature by demonstrating its feasibility in an overburdened health system in Latin America and adopting a protocol in which patients themselves actively sought advice. Mobile phones were found to be a feasible and acceptable means of delivering follow-up care to patients after hospital discharge, including medication and self-care instructions, preventive advice, and linkage services to community care.

\section{LIMITATIONS AND FUTURE DIRECTIONS}

This study had several limitations. First, there was no control group, so patient outcomes cannot be attributed to the intervention. However, as this initial study tested acceptability and feasibility rather than clinical outcomes, there was less need for a control group at this stage. Second, historical data regarding the percentage of patients who typically receive follow-up after hospitalization were not available, as they are not routinely collected. This lack of data was a primary motivation for the design of the current project, a candidate system for tracking patient follow-up in the future.

Finally, the majority of participants were young women with an obstetric condition, many postpartum, reflecting that perinatal conditions account for the majority of admissions in this hospital. Thus, our results may not generalize to a patient cohort experiencing more diverse health conditions. In our sample, new mothers required the most follow-up per patient of all participants. In contrast, some enrolled patients, including those hospitalized for acute injuries or orthopedic surgeries, required little follow-up and are least likely to benefit from a program like this. The current study indicates that mobile phone interventions are a promising avenue for integrated community based maternal-child health interventions. ${ }^{17}$ Future studies will target the mobile phone follow-up program specifically to new mothers, conducting controlled trials of its impact on maternal and child health outcomes.

In summary, this study demonstrated, for the first time, the acceptability and feasibility of a patient-centered mobile phone program to facilitate follow-up care after hospital discharge. The acceptability of the program was demonstrated through high rates of enrollment (89\% of eligible patients enrolled) and self-initiated participation. Its feasibility was demonstrated by the successful provision of follow-up care and by patients' ability and willingness to participate in their own healthcare via mobile phone. The program's addition of nurses specifically charged with ensuring systematic follow-up care that was otherwise absent in standard discharge procedures was certainly one element of the program's success. The success of this program can additionally be attributed to the collaborative manner in which it was developed. The need for improved community-based care following hospitalization was identified by healthcare professionals in Quito. The protocol was jointly devised and implemented by these healthcare professionals and University of Michigan researchers. This collaborative model enables community ownership and facilitates building long-term research capacity for Ecuadorian personnel while working to improve community health. This study represents an important step forward in the evidencebased incorporation of low-cost technologies into ongoing community-based efforts to improve follow-up care and health outcomes in low-resource communities.

\section{Acknowledgments}

The authors thank the Ecuadorian Public Health Ministry for the collaboration of their personnel and use of their facilities. This work was supported in part by the Center for Global Health and The Quito Project of the University of Michigan.

\section{Disclosure Statement}

No competing financial interests exist.

\section{REFERENCES}

1. Kahn J. 'Mobile' health needs and opportunities in developing countries. Health Aff (Millwood) 2010;29:252-258.

2. International Telecommunications Union. The world in 2010, ICT facts and figures. Available at www.itu.int/ITU-D/ict/ (last accessed October 20, 2010).

3. CIA World Factbook: Ecuador. Available at www.cia.gov/library/publications/ the-world-factbook/geos/ec.html (last accessed July 11, 2011).

4. Chen Z, Fang LZ, Chen LY, Dai HL. Comparison of an SMS text messaging and phone reminder to improve attendance at a health promotion center A randomized controlled trial [in Chinese]. J Zhejiang Univ Sci B 2008;9: 34-38. 
5. DaCosta TM, Salomao PL, Martha AS, Pisa IT, Sigulem D. The impact of short message service text messages sent as appointment reminders to patients' cell phones at outpatient clinics in Sao Paulo, Brazil. Int J Med Inform 2009;79: 65-70.

6. Pop-Eleches $\mathrm{C}$, Thirumurthy $\mathrm{H}_{1}$ Habyarimana JP, Zivin JG, Goldstein MP, de Walque $D$, Mackeen $L$, et al. Mobile phone technologies improve adherence to antiretroviral treatment in a resource-limited setting: A randomized controlled trial of text message reminders. AIDS 2011;25:1137.

7. Free C, Phillips G, Felix L, Galli L, Patel V, Edwards P. The effectiveness of Mhealth technologies for improving health and health services: A systematic review protocol. BMC Res Notes 2010;3:250-256.

8. Bali S, Singh AJ. Mobile phone consultation for community healthcare in rural north India. J Telemed Telecare 2007;13:421-424.

9. Kim S, Kim H. Effectiveness of mobile and internet intervention in patients with obese type 2 diabetes. Int J Med Inform 2008;77:399-404.

10. Krishna S, Boren SA, Balas EA. Healthcare via cell phones: A systematic review. Telemed J E Health 2009;15:231-240.

11. Dudas $V$, Bookwalter $T$, Kerr KM, Pantilat SZ. The impact of follow-up telephone calls to patients after hospitalization. Am J Med 2001;111:26S-30S.

12. Mahmud N, Rodriguez J, Nesbit J. Mobiles in Malawi: A text message-based intervention to bridge the patient-physician gap in the rural developing world. Global Pulse 2010;6:1-6.

13. Blaya JA, Fraser HS, Holt B. E-health technologies show promise in developing countries. Health Aff (Millwood) 2010;29:244-251.
14. Rigby M. Impact of telemedicine must be defined in developing countries. BMJ 2002;324:47-48.

15. Curioso WH, Mechael PN. Enhancing 'M-health" with south-to-south collaborations. Health Aff (Millwood) 2010;29:264-267.

16. U.S. Department of State. Background note: Ecuador. Available at www.state .gov/r/pa/ei/bgn/35761.htm (last accessed August 29, 2011).

17. Ekman B, Pathmanathan I, Liljestrand J. Integrating health interventions for women, newborn babies, and children: A framework for action. Lancet 2008; 372:990-1000.

Address correspondence to: Sophia Merajver, M.D., Ph.D. Center for Global Health University of Michigan

1214 S. University Ave. $2^{\text {nd }}$ Floor, Suite $C$

Ann Arbor, MI 48104-2592

E-mail: smerajve@med.umich.edu

Received: August 3, 2011

Revised: September 9, 2011

Accepted: September 12, 2011 